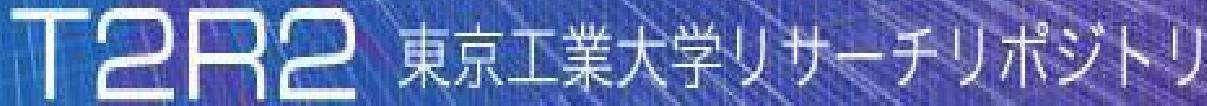

\section{Tokyo Tech Research Repository}

\section{論文 /著書情報 \\ Article /Book Information}

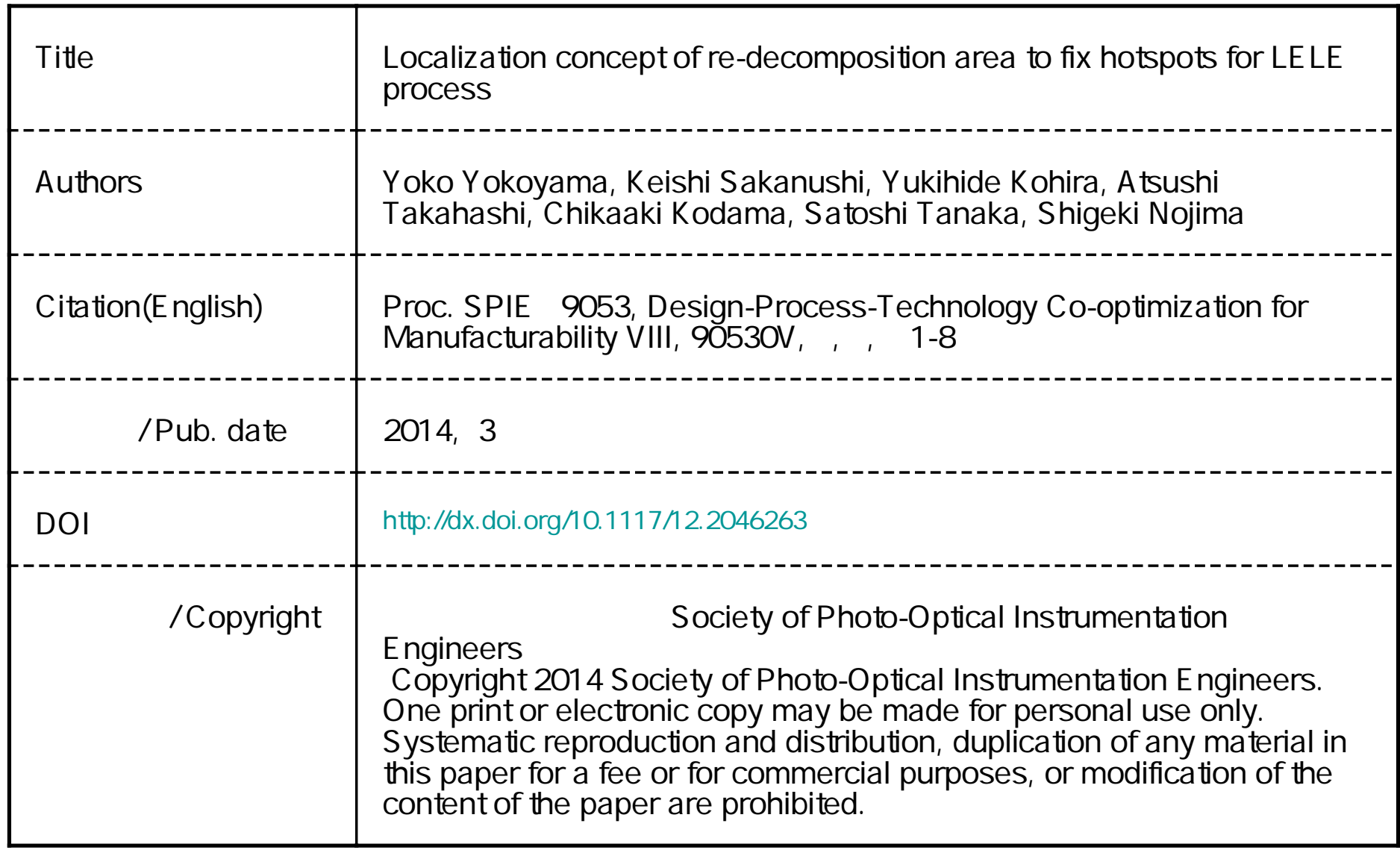




\title{
Localization concept of re-decomposition area to fix hotspots for LELE process
}

\author{
Yoko Yokoyama $^{* a}$, Keishi Sakanushi ${ }^{* * a}$, Yukihide Kohira $^{b}$, Atsushi Takahashi ${ }^{c}$, \\ Chikaaki Kodama ${ }^{a}$, Satoshi Tanaka ${ }^{a}$ and Shigeki Nojima ${ }^{a}$ \\ ${ }^{a}$ Toshiba Corporation, Yokohama, Japan \\ ${ }^{b}$ The University of Aizu, Aizu-Wakamatsu, Japan \\ ${ }^{c}$ Tokyo Institute of Technology, Tokyo, Japan
}

\begin{abstract}
Among several double patterning methods, Litho-Etch-Litho-Etch type DPT is known to have an advantage of layout flexibility. There are two problems when a hotspot, which is not fixable by tuning OPC, is detected on a wafer or during lithography compliance verification. One is to redo decomposition, OPC and verification is quite time consuming. The other is a risk to introduce a new hotspot at different locations. In this report, a new method to fix hotspot with layout modification of limited area will be presented. The proposed method can reduce not only repair turnaround time but also a risk of new hotspot generation.
\end{abstract}

Keywords: Double patterning, DFM, LELE, layout modification, hotspot, decomposition

\section{INTRODUCTION}

It has been a long time since single patterning with an ArF immersion exposure tool reached its physical limit. Double patterning technology (DPT) is one of the promising techniques for $20 \mathrm{~nm}$ technology node and beyond. ${ }^{1}$ For Litho-EtchLitho-Etch (LELE) process, which is one of the DPTs, layout design needs to be decomposed into two masks, usually utilizing graph theory. ${ }^{2-4}$ Using decomposed two masks, lithography and etching process are proceeded twice respectively. Comparing other types of DPTs, LELE type DPT is known to have an advantage of layout flexibility. ${ }^{5}$

Even with matured design rules, OPC and process recipe, there is always a risk of hotspots especially for advanced technology nodes. ${ }^{6}$ There are two problems when a hotspot, which is not fixable by tuning OPC, is detected on a wafer or during verification by lithography simulation for a decomposed mask. One is repeating a data preparation flow including decomposition, OPC and verification by lithography simulation is quite time consuming. This is because even small layout modification may cause influence layout decomposition over a whole area. The other is a risk to introduce new hotspots at different locations. After re-decomposition of layout to fix detected hotspots, brand new hotpots may be detected where there is initially no hotspot, which makes it very difficult to completely eliminate hotspots. Considering two problems mentioned above, conventional concept of hotspot fixing is inefficient and a new way to solve the problems are required.

In this research, a new localization concept of re-decomposition area to fix hotspots for LELE process is introduced, which can avoid two kinds of risks mentioned above. Although layout needs to be modified whenever hotspots are detected, this work is trying to localize re-decomposition area by focusing on the spaces between two patterns on a same mask since it reduces hotspot fixing turnaround time efficiently.

\footnotetext{
*yoko.yokoyama@mail.semicon.toshiba.co.jp

** keishi.sakanushi@toshiba.co.jp 


\section{MASK DATA PREPAREATION FLOW FOR LELE}

In Litho-Etch-Litho-Etch (LELE) process, layout is decomposed into 2 masks, usually by solving graph coloring problem. Fig. 1 shows a schematic diagram for LELE mask data preparation (MDP) flow. In this case, layout is decomposed to mask A and mask B. Then OPC will be applied to post decomposed layouts. After OPC, lithography compliance of each mask will be verified as well as overlapped images. If no error is detected in lithography compliance check (LCC), hotspot clean mask A and mask B are taped out to the mask house.

If there are any hotspots detected in LCC, hotspot needs to be fixed by modifying layout. After modifying layout, it is necessary to back to the first step and repeat the same flow, including decomposition, OPC and LCC steps. Optical simulation such as OPC and LCC is known to be very time consuming and decomposition is also a time consuming step. Therefore, fixing hotpot with layout modification could take quite long time. Some iteration which consists of those time consuming steps may be required to obtain hotspot clean masks. In LELE process, minimizing optical simulation area and graph coloring area is the most important issue to reduce MDP time.

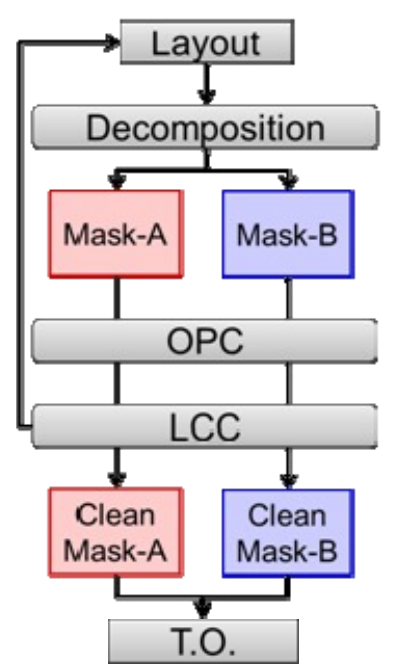

Figure 1: A flow chart of mask data preparation flow for LELE process

\section{LAYOUT MODIFICATION AND HOTSPOT FIXING FOR LELE}

In LELE process, a layout will be decomposed into two masks after a decomposition step. In the case of Fig 2, the layout shown in Fig. 2(a) is decomposed to Mask A and Mask B and a hotspot is detected during LCC step on mask A as shown in Fig. 2(b). To fix this hotspot, layout needs to be modified. For example, layout after modification could be as shown in Fig. 2(c). The modified layout after decomposition of Fig. 2(c) needs to be decomposed again since layout was changed. After modification, new Mask A and new Mask B are shown in Fig 2(d). In this case, new Mask A and original Mask A are different, and New Mask B and original Mask B are also different. Each combination turns out to be different. Since the new two masks are entirely different from old masks, LCC needs to be done again for both two masks even though a hotspot is detected in only one mask.

Now, the hotspot on Mask A is successfully fixed as shown in Fig. 2(d). However, new hotspots are possibly generated at different location - in this case on new Mask B. Obviously, the layout should be modified again to fix this new generated hotspot. There is still a possibility to detect new hotspots. In the worst case scenario, infinite iteration of decomposition, OPC and LCC might be required. 


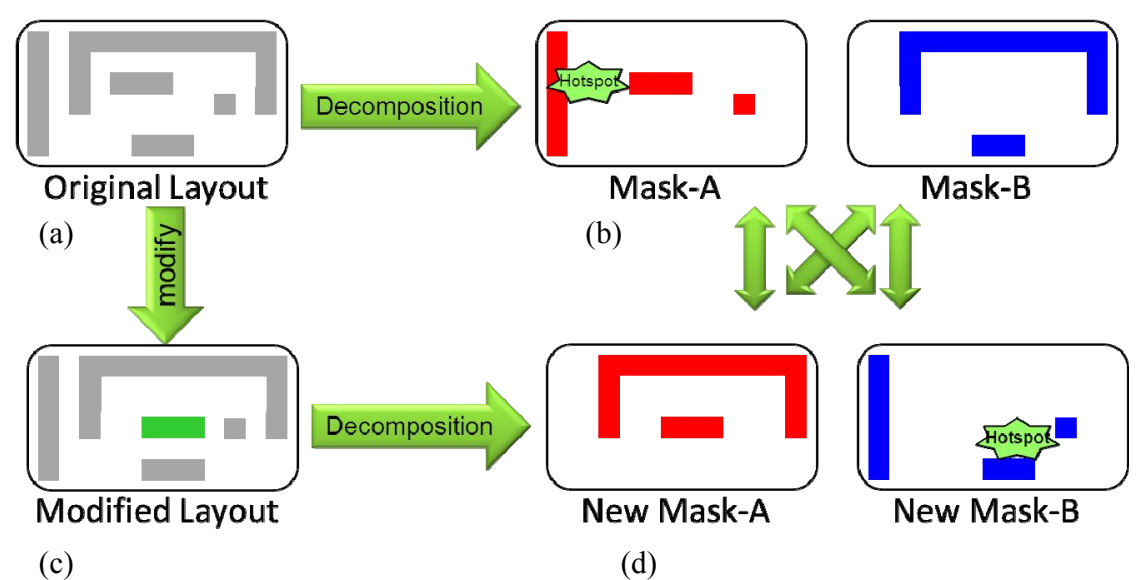

Figure 2: Layout modification example to fix a hotspot

As discussed above, there are problems to fix hotspots in LELE process. Even though layout is modified locally, pattern assignment on large area is influenced. As a result, decomposition, OPC and LCC steps of whole area may need to be repeated. The worst part of it is that hotspot fixing may not be converged since new hotspots are possibly generated.

One of this research's goals is to reduce computation time to fix hotspot. We also try to prevent new hotspot generation due to layout modification. The key point here is to minimize the areas of re-decomposition, OPC and LCC, which are induced by hotspot fixing. In the next section, a new proposed hotspot modification flow for LELE process, which minimizes re-decomposition, OPC and LCC area, will be introduced.

\section{HOTSPOT FIXING FLOW FOR LELE DPT}

Fig. 3 is a flow chart of the proposed hotspot fixing for LELE double patterning. The design layout is decomposed into two masks, which are mask A and mask B. Then, OPC and LCC are applied to both masks. If there is no hotspot, both masks are released to the mask house. When a hotspot is detected and if the detected hotspot cannot be fixed by tuning $\mathrm{OPC}$, layout modification is required. However, the proposed flow chat can avoid repeating the whole flow.

\subsection{Concept of the Proposed Hotspot Fixing Method}

The proposed method is to define and extract re-decomposition area around a detected hotspot. Since the layout for the rest of the area remains the same, no re-decomposition is necessary as well as OPC and LCC. Re-decomposition is required only for the extracted area. After re-decomposition, sub mask A and sub mask B are obtained. OPC and LCC are also required for generated sub masks. Although this flow needs to be repeated until no hotspot is detected, the risk of generating new hotspots becomes low since the area of sub-masks is limited.

If no hotspot is detected during LCC, the sub mask A and sub mask B are merged with the other area, and then masks are ready to be released. The essential point of this proposed flow is how we can define and extract its redecomposition area. Before discussing how we can define the re-decomposition area, it is important to understand under which conditions, the patterns should be changed their colors. 


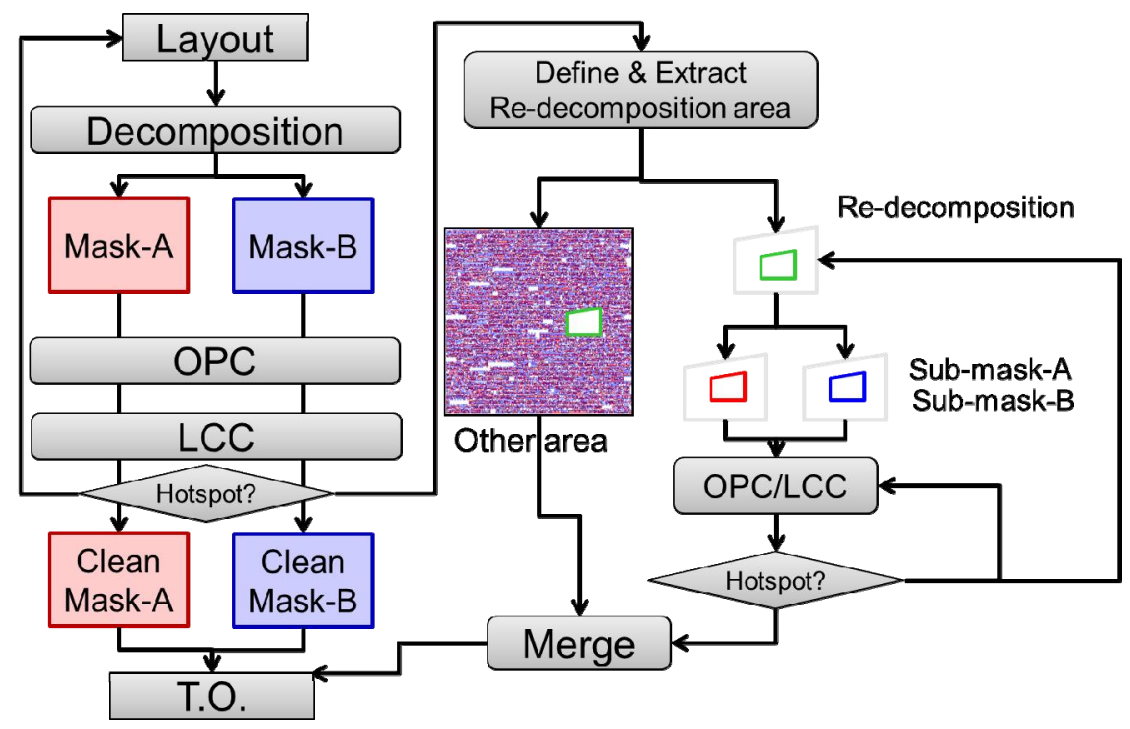

Figure 3: A proposed hotspot fixing flow

\subsection{Condition of Color Change}

In order to understand what situation affects to the color change, we review the relation between two patterns before thinking large layout. For LELE process, the relations between two patterns are classified into three categories by two kinds of distances between them, which are L1 and L2. L1 is the different color minimum space and this is determined by process assumption like overlay. L2 is the same color minimum space, which is determined by lithographic resolution. Usually, L2 is longer than L1.

Fig. 4 shows a whole variation of relations between two patterns, which are pattern A' and pattern B. In this case, A' is the pattern after layout modification and B is the pattern near pattern $A^{\prime}$. If the distance between these two patterns is larger than L1 but less than L2, the color of either of patterns needs to be changed, since the space is less than the minimum space rule for same colored patterns, which is L2. When the distance between two patterns is larger than L2, patterns can remain the same color. In the case that the distance is less than L1, layouts are under the design rule (DR) violation, which means those types of layout modification are prohibited.

The key issue here is that it is required to pay attention to this relation in order to control the re-decomposition area. If there is this kind of situation, we need to search for the area which needs color change and re-decomposition. In the next section, the way to define the re-decomposition area, by focusing on the space between the same colored patterns, is discussed.

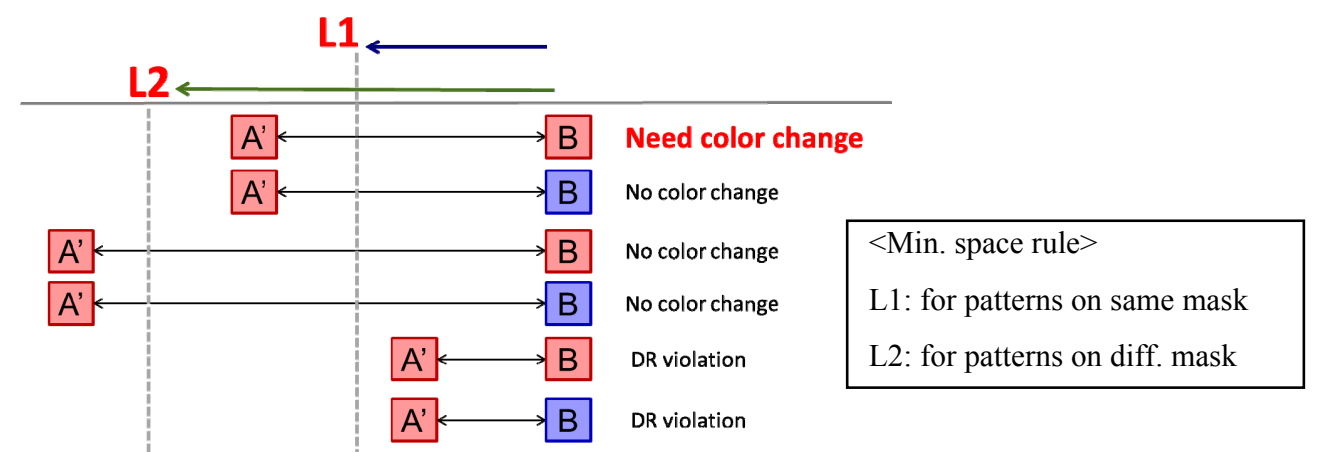

Figure 4: Minimum space rules and pattern relations 


\section{DEFINITION OF RE-DECOMPOSITION AREA}

In this section, step-by-step explanation how to define the re-decomposition area using a very simple example case is described. Step0 in Fig. 5(a) shows a layout before modification. Red patterns are on mask A and blue patterns are on mask B. It is assumed that there is a hotspot detected as shown in Fig 5(a) and this hotspot needs to be fixed. As shown in Step1, in this case layout is modified and pattern A moves to pattern A', which is red color on mask A. In Step2, we focus on red colored patterns since in a previous section it is shown that only same colored patterns within minimum space rules affect color changes. Each red colored polygon is expanded by a half of L2 as shown in Fig. 5(c). In other words; each polygon is given a territory with the expansion of a half of L2.

Step3 is to check whether any overlap exists or not for generated regions in step 2. If there is no overlapped area, none of the patterns needs color change. That means this modification does not require re-decomposition. In this case, OPC and LCC need only a region around modified pattern A'. On the other hand, if there is any overlapped area, some patterns need color change, which means re-decomposition is required in this case and requires moving to step 4 .

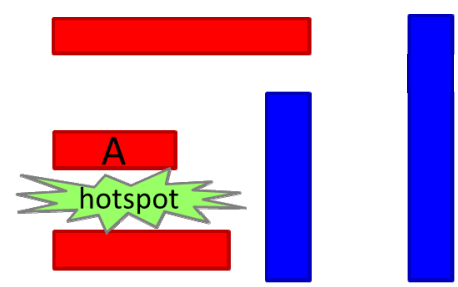

Step0: Layout before modification

(a)

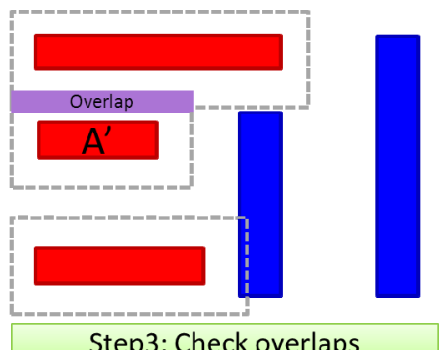

Step3: Check overlaps

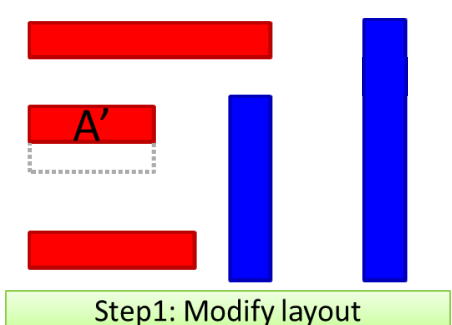

(b)

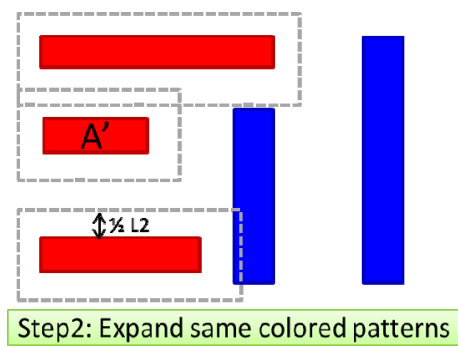

(c)

(d)

Figure 5: Step-by-step description of re-decomposition area definition

In step 4, the area enlarged by a half of L2 is generated for all polygons including red patterns and blue patterns as shown in Fig. 6(a). In step 5, the connecting area of generated polygons in step 4, which contains overlapped region and defined as green boundary in Fig 6(b), is extracted. The extracted area in step 5 is the re-decomposition area. In other word, all polygons within this green boundary, which is yellow colored patterns in Fig. 6(c), need to be decomposed again. A blue polygon in Fig. 6(c) is out of green boundary, so this polygon can stay the same color which is blue.

Finally, the re-OPC and re-LCC area are defined in step 7. As shown in Fig. 6(d), the re-decomposition area, which is defined by green boundary, is enlarged the by the optical diameter, which becomes like the purple square. The area defined with purple boundary is for re-OPC and re-LCC. 


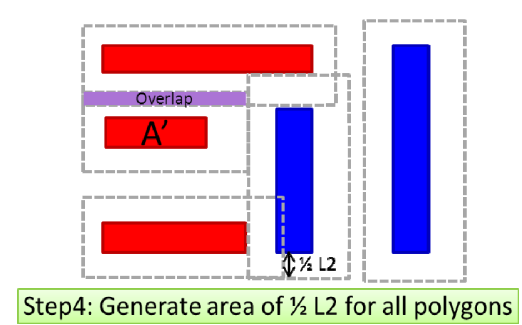

(a)

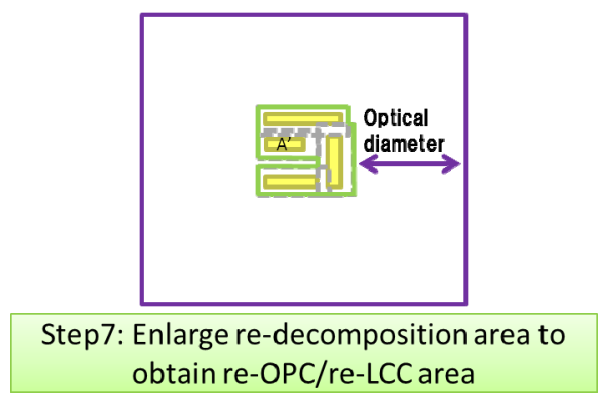

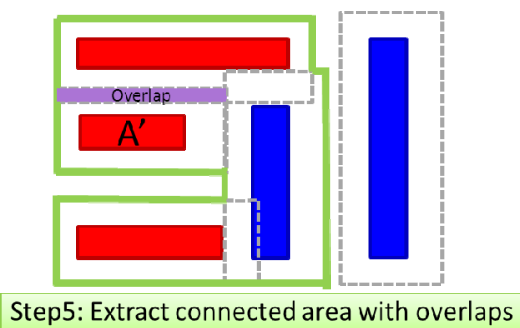

(b)

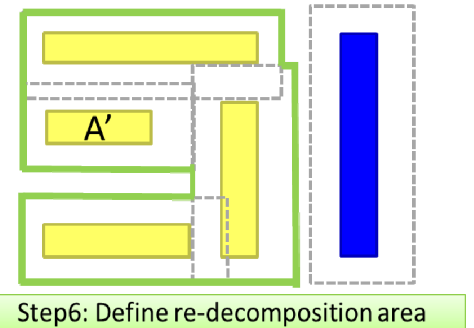

(c)

(d)

Figure 6: Step-by-step description of re-decomposition area definition (continued)

\section{VERIFICATION WITH A TESTCASE}

We prepared the layout from open cell library ${ }^{7}$, which originally had $65 \mathrm{~nm}$ as minimum line and space width. The layout was shrunk to obtain the layout which is equivalent to $20 \mathrm{~nm}$ technology node. Next, layout was modified for LELE process since the original layout was not decomposition compliant. The size of a test case is approximately $30 \mathrm{um} \times 30 \mathrm{um}$. The layout was decomposed into two masks, mask A which was red color and mask B which was blue color. In this test case, we assumed there was a hotspot and a part of layout needed to be modified to fix hotspot.

Fig. 7(b) shows the extracted layout around the hotspot from the sample case. Fig. 7(c) shows an example layout after layout modification. In this case the red polygon at the tip of an arrow was shifted to left. The space between the shifted red polygon and neighboring red polygon violates the minimum space rule for the same colored pattern in this case, which means some of the polygons need to be changed their colors. In other words, re-decomposition is required. In this test case, we tried to fix this single hotspot using our method. Next, re-decomposition area was extracted by using proposed method. Since a pattern on a red mask was changed, we focus on overlaps of the territory of red colored patterns.

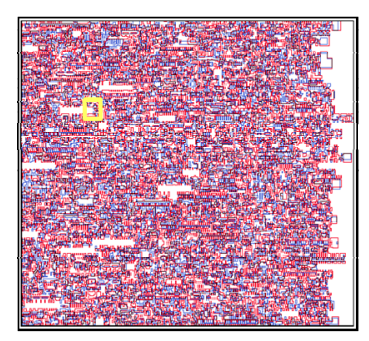

(a)

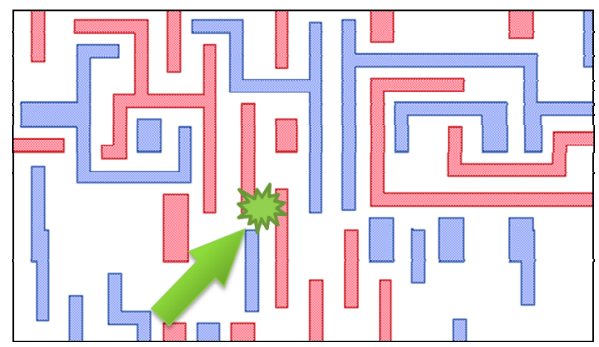

(b)

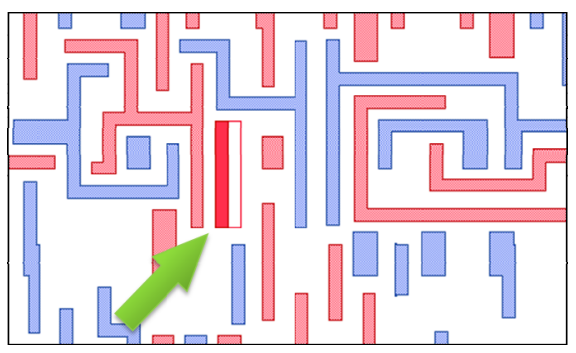

(c)

Figure 7: A test case of the experiment (a), layout around hotspot (b), and layout after modification (c) 
The green color which is shown in Fig.8 is the extracted area as re-decomposition area. As explained in previous sections, we need decomposition only within the extracted area. Finally, we expanded re-decomposition area by optical diameter to obtain re-OPC are re-LCC area. As we can see, our proposed method could limit the area for redecomposition, re-OPC and re-LCC area.

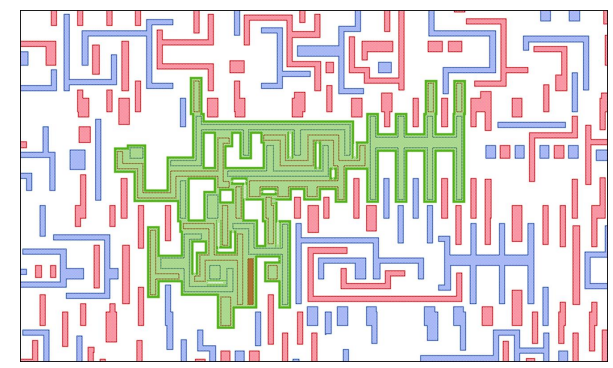

(a)

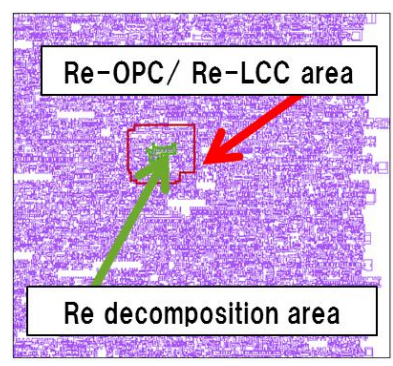

(b)

Figure 8: (a), defined re-decomposition area and (b) re-OPC/LCC area of the test case

Fig. 9 is the result of our case study. Y-axis shows a percentage of area, where total area of test case is set to $100 \%$. Grey bar is the area for using conventional way to fix layout. Green bar is the area for using our proposed method. Left bars show the area for re-decomposition. In a conventional way, we needed to decompose a whole layout to fix hotspot, since we did not have a method to know in which area the coloring influence was occurred to. On the other hand, with our proposed method, the area became less than $1 \%$ of conventional one since we tried to minimize and define the area of re-decomposition. The right bars are the comparison of OPC and LCC when the layout was modified to fix hotspot. OPC and LCC require larger area then re-decomposition since the optical diameter should be taken into account. Even with the consideration of optical diameter, the required area for re-OPC and re-LCC with our proposed method was approximately only $4 \%$ of the total area. OPC and LCC involve optical simulation, which means they are very time consuming steps. Since calculation time of such optical simulation is related to the area of calculation, the total modification time become much smaller.

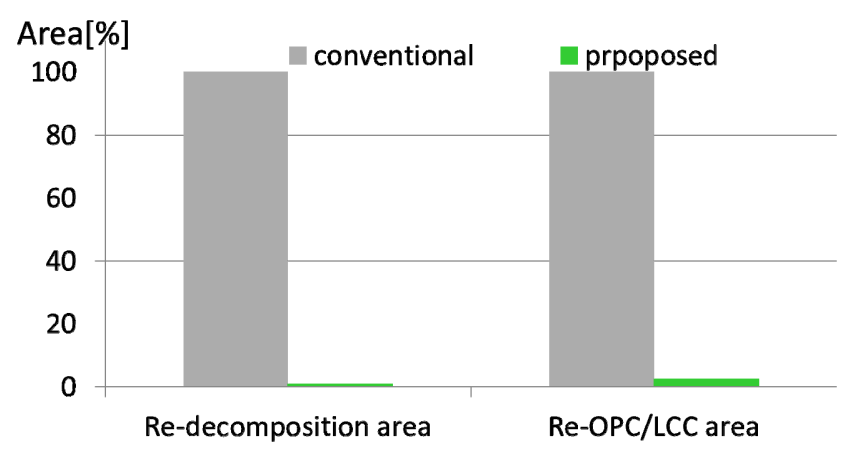

Figure 9: Results of a test study

\section{CONCLUSION}

In this research, a new method to localize re-decomposition area to fix hotspots for LELE process is presented. For LELE process, there are two main issues on fixing hotspot for LELE - larger area of re-decomposition and infinite iteration of hotspot fixing steps. We introduce the way to localize the re-decomposition area by focusing on same colored 
patterns after layout modification. With a test case, we succeeded to significantly reduce the area of re-decomposition and OPC/LCC area with our proposed method. The proposed method can reduce not only repair turnaround time but also a risk of new hotspot generation. As for the future work, we will apply this method in order to find the most effective way to fix hotspots for double patterning.

\section{REFERENCES}

[1] Bailey, G. E., Tritchkov, A., Park, J.-W., Hong, L., Wiaux, V., Hendrickx, E., Verhaegen, S., Xie, P., and Versluijs, J., "Double pattern EDA solutions for 32nm HP and beyond," in [Proc. SPIE], 6521, 65211K (2007).

[2] Kahng, A., Park, C.-H., Xu, X., and Yao, H., "Layout decomposition for double patterning lithography," in [Proc. ICCAD], 465-472 (2008).

[3] Ghaida, R. S., Agarwal, K. B., Nassif, S. R., Yuan, X., Liebmann, L. W., and Gupta, P., “A framework for double patterning-enabled design," in [Proc. ICCAD], 14-20 (2011).

[4] Ghaida, R. S., Agarwal, K. B., Nassif, S. R., Yuan, X., Liebmann, L. W., and Gupta, P., "Layout decomposition and legalization for double-patterning technology,” IEEE Trans. Comput.-Aided Des. Integr. Circuits Syst. 32, 202-215 (2 2013).

[5] Arisa Hara, Eiichi Nishimura, Masato Kushibiki, Shoichi Yamauchi, Sakurako Natori, Kazuo Yabe, Kenichi Oyama, Hidetami Yaeasghi, " Advanced self-aligned DP process development for 22-nm node and beyond" in [Proc. SPIE], 7636, 76391T (2010)

[6] Sachiko Kobayashi, Suigen Kyoh, Toshiya Kotani, Yoko Takekawa, Soichi Inoue, and Koji Nakamae, "Full-Chip Layout Optimization for Process Margin Enhancement Using Model-Based Hotspot Fixing System” Jpn. J. Appl. Phys. 49, 06GB02 (2010)

[7] “Nangate Open Cell Library.” http://www.si2.org/openeda.si2.org/projects/nangatelib. 